\title{
Deep Learning Identity-Preserving Face Space
}

\author{
Zhenyao Zhu ${ }^{1, *} \quad$ Ping Luo ${ }^{1,3, *} \quad$ Xiaogang Wang ${ }^{2} \quad$ Xiaoou Tang ${ }^{1,3, \dagger}$ \\ ${ }^{1}$ Department of Information Engineering, The Chinese University of Hong Kong \\ ${ }^{2}$ Department of Electronic Engineering, The Chinese University of Hong Kong \\ ${ }^{3}$ Shenzhen Institutes of Advanced Technology, Chinese Academy of Sciences
}

zz012@ie.cuhk.edu.hk pluo.lhi@gmail.com

xgwang@ee.cuhk.edu.hk xtang@ie.cuhk.edu.hk

\begin{abstract}
Face recognition with large pose and illumination variations is a challenging problem in computer vision. This paper addresses this challenge by proposing a new learningbased face representation: the face identity-preserving (FIP) features. Unlike conventional face descriptors, the FIP features can significantly reduce intra-identity variances, while maintaining discriminativeness between identities. Moreover, the FIP features extracted from an image under any pose and illumination can be used to reconstruct its face image in the canonical view. This property makes it possible to improve the performance of traditional descriptors, such as LBP [2] and Gabor [31], which can be extracted from our reconstructed images in the canonical view to eliminate variations. In order to learn the FIP features, we carefully design a deep network that combines the feature extraction layers and the reconstruction layer. The former encodes a face image into the FIP features, while the latter transforms them to an image in the canonical view. Extensive experiments on the large MultiPIE face database [7] demonstrate that it significantly outperforms the state-of-the-art face recognition methods.
\end{abstract}

\section{Introduction}

In many practical applications, the pose and illumination changes become the bottleneck for face recognition [36]. Many existing works have been proposed to account for such variations. The pose-invariant methods can be generally separated into two categories: 2D-based [17, 5, 23] and 3D-based [18, 3]. In the first category, poses are either handled by $2 \mathrm{D}$ image matching or by encoding a test image using some bases or exemplars. For example,

\footnotetext{
${ }^{*}$ indicates equal contribution.

${ }^{\dagger}$ This work is supported by the General Research Fund sponsored by the Research Grants Council of the Kong Kong SAR (Project No. CUHK 416312 and CUHK 416510) and Guangdong Innovative Research Team Program (No.201001D0104648280).
}

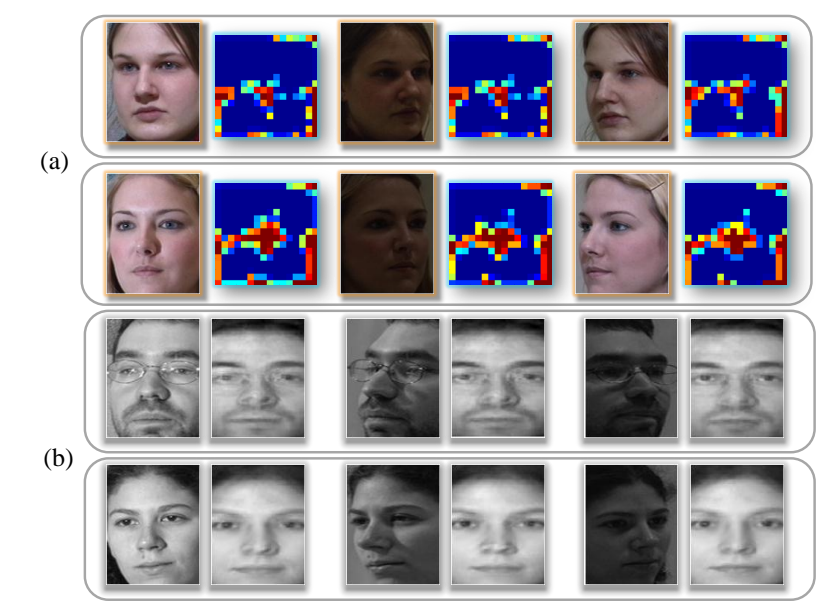

Figure 1. Three face images under different poses and illuminations of two identities are shown in (a). The FIP features extracted from these images are also visualized. The FIP features of the same identity are similar, although the original images are captured in different poses and illuminations. These examples indicate that FIP features are sparse and identity-preserving (blue indicates zero value). (b) shows some images of two identities, including the original image (left) and the reconstructed image in the canonical view (right) from the FIP features. The reconstructed images remove the pose and illumination variations and retain the intrinsic face structures of the identities. Best viewed in color.

Carlos et al. [5] used stereo matching to compute the similarity between two faces. Li et al. [17] represented a test face as a linear combination of training images, and utilized the linear regression coefficients as features for face recognition. 3D-based methods usually capture 3D face data or estimate $3 \mathrm{D}$ models from $2 \mathrm{D}$ input, and try to match them to a $2 \mathrm{D}$ probe face image. Such methods make it possible to synthesize any view of the probe face, which makes them generally more robust to pose variation. For instance, Li et al. [18] first generated a virtual view for the probe face by using a set of 3D displacement fields sampled from a 3D face database, and then matched the synthesized face with the gallery faces. Similarly, Asthana et al. [3] matched the $3 \mathrm{D}$ model to a $2 \mathrm{D}$ image using the view-based active appearance model.

The illumination-invariant methods [26, 17] typically 

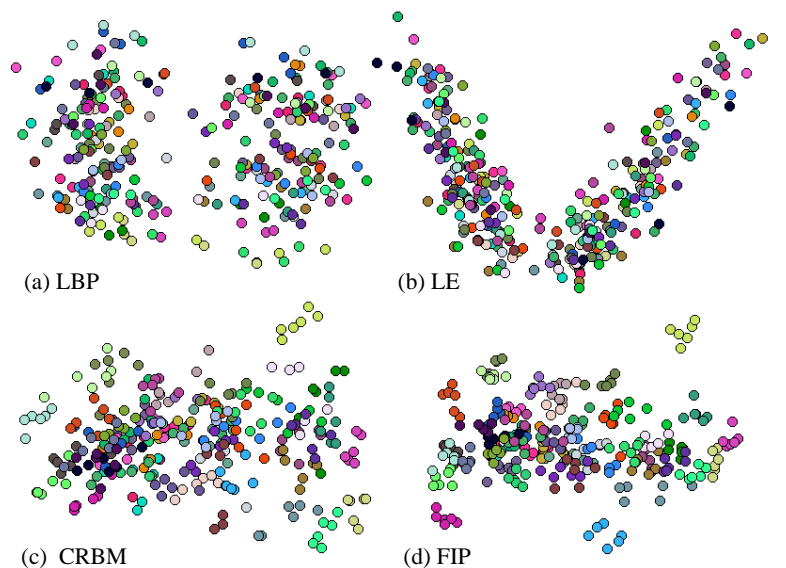

Figure 2. The LBP (a), LE (b), CRBM (c), and FIP (d) features of 50 identities, each of which has 6 images in different poses and illuminations are projected into two dimensions using Multidimensional scaling (MDS). Images of the same identity are visualized in the same color. It shows that FIP has the best representative power. Best viewed in color.

make assumptions about how illumination affects the face images, and use these assumptions to model and remove the illumination effect. For example, Wagner et al. [26] designed a projector-based system to capture images of each subject in the gallery under a few illuminations, which can be linearly combined to generate images under arbitrary illuminations. With this augmented gallery, they adopted sparse coding to perform face recognition.

The above methods have certain limitations. For example, capturing 3D data requires additional cost and resources [18]. Inferring 3D models from 2D data is an illposed problem [23]. As the statistical illumination models [26] are often summarized from controlled environment, they cannot be well generalized in practical applications.

In this paper, unlike previous works that either build physical models or make statistical assumptions, we propose a novel face representation, the face identitypreserving (FIP) features, which are directly extracted from face images with arbitrary poses and illuminations. This new representation can significantly remove pose and illumination variations, while maintaining the discriminativeness across identities, as shown in Fig 1 (a). Furthermore, unlike traditional face descriptors, e.g. LBP [2], Gabor [31], and LE [4], which cannot recover the original images, the FIP features can reconstruct face images in the frontal pose and with neutral illumination (we call it the canonical view) of the same identity, as shown in Fig 1 (b). With this attractive property, the conventional descriptors and learning algorithms can utilize our reconstructed face images in the canonical view as input so as to eliminate the negative effects from poses and illuminations.

Specifically, we present a new deep network to learn the FIP features. It utilizes face images with arbitrary pose and illumination variations of an identity as input, and reconstructs a face in the canonical view of the same identity as the target (see Fig 3. First, input images are encoded through feature extraction layers, which have three locally connected layers and two pooling layers stacked alternately. Each layer captures face features at a different scale. As shown in Fig 3 , the first locally connected layer outputs 32 feature maps. Each map has a large number of high responses outside the face region, which mainly capture pose information, and some high responses inside the face region, which capture face structures (red indicates large response and blue indicates no response). On the output feature maps of the second locally connected layer, high responses outside the face region have been significantly reduced, which indicates that it discards most pose variations while retain the face structures. The third locally connected layer outputs the FIP features, which is sparse and identity-preserving.

Second, the FIP features recover the face image in the canonical view using a fully-connected reconstruction layer. As there are large amount of parameters, our network is hard to train using tranditional training methods [14, 12]. We propose a new training strategy, which contains two steps: parameter initialization and parameter update. First, we initialize the parameters based on the least square dictionary learning. We then update all the parameters by back-propagating the summed squared reconstruction error between the reconstructed image and the ground truth.

Existing deep learning methods for face recognition are generally in two categories: (1) unsupervised learning features with deep models and then using discriminative methods (e.g. SVM) for classification [21, 10, 15]; (2) directly using class labels as supervision of deep models [6, 24]. In the first category, features related to identity, poses, and lightings are coupled when learned by deep models. It is too late to rely on SVM to separate them later. Our supervised model makes it possible to discard pose and lighting features from the very bottom layer. In the second category, a ' $0 / 1$ ' class label is a much weaker supervision, compared with ours using a face image (with thousands of pixels) of the canonical view as supervision. We require the deep model to fully reconstruct the face in the canonical view rather than simply predicting class labels, and this strong regularization is more effective to avoid overfitting. This design is suitable for face recognition, where a canonical view exists. Different from convolutional neural networks whose filters share weights, our filers are localized and do not share weights since we assume different face regions should employ different features.

This work makes three key contributions. (1) We propose a new deep network that combines the feature extraction layers and the reconstruction layer. Its architecture is carefully designed to learn the FIP features. These features can eliminate the poses and illumination variations, and 


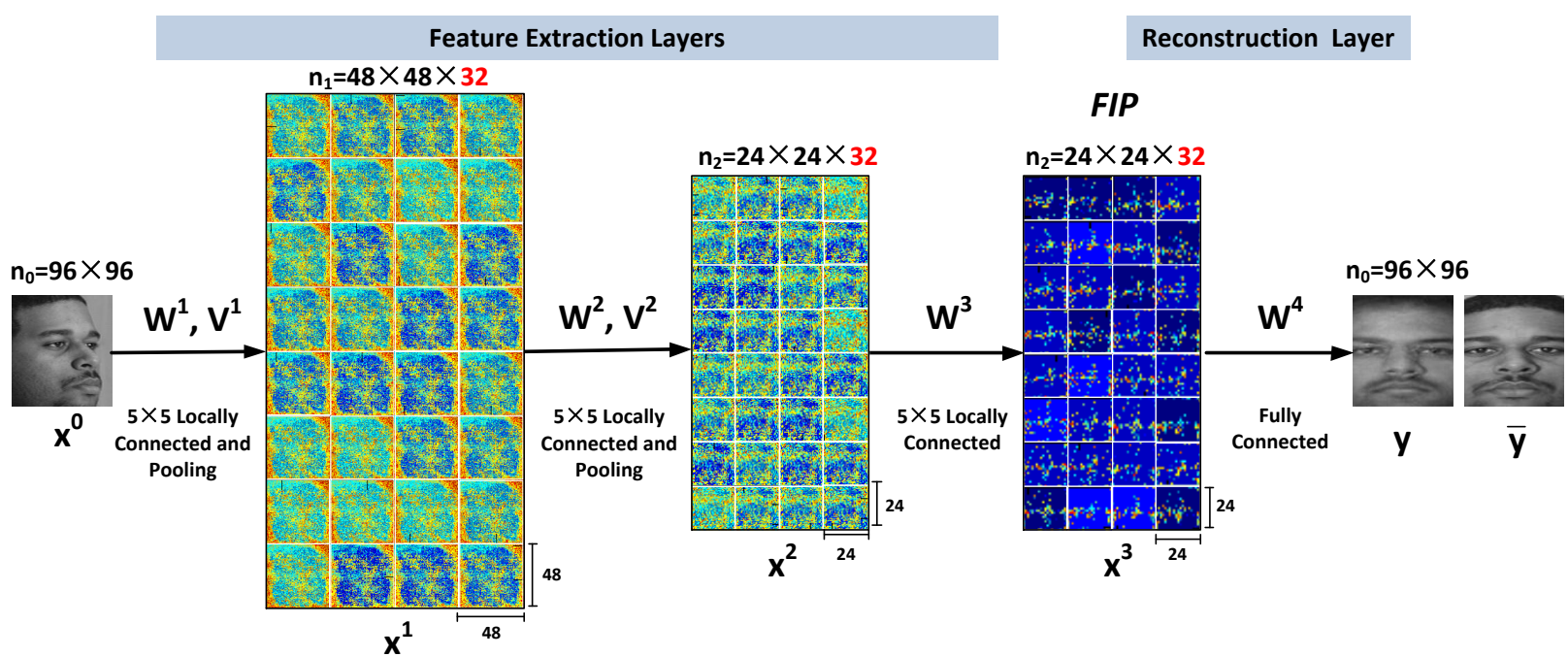

Figure 3. Architecture of the deep network. It combines the feature extraction layers and reconstruction layer. The feature extraction layers include three locally connected layers and two pooling layers. They encode an input face $\mathbf{x}^{0}$ into FIP features $\mathbf{x}^{3} \cdot \mathbf{x}^{1}, \mathbf{x}^{2}$ are the output feature maps of the first and second locally connected layers. FIP features can be used to recover the face image $\mathbf{y}$ in the canonical view. $\overline{\mathbf{y}}$ is the ground truth. Best viewed in color.

maintain discriminativeness between different identities. (2) Unlike conventional face descriptors, the FIP features can be used to reconstruct a face image in the canonical view. We also demonstrate significant improvement of the existing methods, when they are applied on our reconstructed face images. (3) Unlike existing works that need to know the pose of a probe face, so as to build models for different poses specifically, our method can extract the FIP features without knowing information on pose and illumination. The FIP features outperform the state-of-the-art methods, including both 2D-based and 3D-based methods, on the MultiPIE database [7].

\section{Related Work}

This section reviews related works on learning-based face descriptors and deep models for feature learning.

Learning-based descriptors. Cao et al. [4] devised an unsupervised feature learning method (LE) with randomprojection trees and PCA trees, and adopted PCA to gain a compact face descriptor. Zhang et al. [35] extended [4] by introducing an inter-modality encoding method, which can match face images in two modalities, e.g. photos and sketches, significantly outperforming traditional methods [25, 30]. There are studies that learn the filters and patterns for the existing handcrafted descriptors. For example, Guo et al. [8] proposed a supervised learning approach with the Fisher separation criterion to learn the patterns of LBP [2]. Zhen et al. [16] adopted a strategy similar to LDA to learn the filters of LBP. Our FIP features are learned with a multi-layer deep model in a supervised manner, and have more discriminative and representative power than the above works. We illustrate the feature space of FIP compared with LE [4] and LBP [2] in Fig 2](a), (b) and (d), respectively, which show that the FIP space better maintains both the intra-identity consistency and the inter-identity discriminativeness.

Deep models. The deep models learn representations by stacking many hidden layers, which are layer-wisely trained in an unsupervised manner. For example, the deep belief networks [9] (DBN) and deep Boltzmann machine [22] (DBM) stack many layers of restricted Boltzmann machines (RBM) and can extract different levels of features. Recently, Huang et al. [10] introduced the convolutional restricted Boltzmann machine (CRBM), which incorporates local filters into RBM. Their learned filters can preserve the local structures of data. Sun et al. [24] proposed a hybrid Convolutional Neural Network-Restricted Boltzmann Machine (CNN-RBM) model to learn relational features for comparing face similarity. Unlike DBN and DBM employ fully connected layers, our deep network combines both locally and fully connected layers, which enables it to extract both the local and global information. The locally connected architecture of our deep network is similar to CRBM [10], but we learn the network with a supervised scheme and the FIP features are required to recover the frontal face image. Therefore, this method is more robust to pose and illumination variations, as shown in Fig 2 (d).

\section{Network Architecture}

Fig 3 shows the architecture of our deep model. The input is a face image $x^{0}$ under an arbitrary pose and illumination, and the output is a frontal face image under neutral illumination $y$. They both have $n_{0}=96 \times 96=$ 9216 dimensions. The feature extraction layers have three 
locally connected layers and two pooling layers, which encode $x^{0}$ into FIP features $x^{3}$.

In the first layer, $x^{0}$ is transformed to 32 feature maps through a weight matrix $W^{1}$ that contains 32 sub-matrices $\left.W^{1}=\left[W_{1}^{1} ; W_{2}^{1} ; \ldots ; W_{32}^{1}\right], \forall W_{i}^{1} \in \mathbb{R}^{n_{0}, n_{0}}\right]$, each of which is sparse to retain the locally connected structure [13]. Intuitively, each row of $W_{i}^{1}$ represents a small filter centered at a pixel of $x^{0}$, so that all of the elements in this row equal zeros except for the elements belonging to the filter. As our weights are not shared, the non-zero values of these rows are not the sam ${ }^{2}$. Therefore, the weight matrix $W^{1}$ results in 32 feature maps $\left\{x_{i}^{1}\right\}_{i=1}^{32}$, each of which has $n_{0}$ dimensions. Then, a matrix $V^{1}$, where $V_{i j} \in\{0,1\}$ encodes the 2D topography of the pooling layer [13], downsamples each of these feature map to $48 \times 48$ in order to reduce the number of parameters need to be learned and obtain more robust features. Each $x_{i}^{1}$ can be computed as ${ }^{3}$

$$
x_{i}^{1}=V^{1} \sigma\left(W_{i}^{1} x^{0}\right),
$$

where $\sigma(x)=\max (0, x)$ is the rectified linear function [19] that is feature-intensity-invariant. So it is robust to shape and illumination variations. $x^{1}$ can be obtained by concatenating all the $x_{i}^{1} \in \mathbb{R}^{48 \times 48}$ together, obtaining a large feature map in $n_{1}=48 \times 48 \times 32$ dimensions.

In the second layer, each $x_{i}^{1}$ is transformed to $x_{i}^{2} 32$ submatrices $\left\{W_{i}^{2}\right\}_{i=1}^{32}, \forall W_{i}^{2} \in \mathbb{R}^{48 \times 48,48 \times 48}$,

$$
x_{i}^{2}=\sum_{j=1}^{32} V^{2} \sigma\left(W_{j}^{2} x_{i}^{1}\right),
$$

where $x_{i}^{2}$ is down-sampled using $V^{2}$ to $24 \times 24$ dimensions. $\mathrm{Eq} 2$ means that each small feature map in the first layer is multiplied by 32 sub-matrices and then summed together. Here, each sub-matrix has sparse structure as discussed above. We can reformulate Eq2 into a matrix form

$$
x^{2}=V^{2} \sigma\left(W^{2} x^{1}\right),
$$

where $W^{2}=\left[W_{1}^{2^{\prime}} ; \ldots ; W_{32}^{2^{\prime}}\right], \forall W_{i}^{2^{\prime}} \in \mathbb{R}^{48 \times 48, n_{1}}$ and $x^{1}=\left[x_{1}^{1} ; \ldots ; x_{32}^{1}\right] \in \mathbb{R}^{n_{1}}$, respectively. $W_{i}^{2^{\prime}}$ is simply obtained by repeating $W_{i}^{2}$ for 32 times. Thus, $x^{2}$ has $n_{2}=24 \times 24 \times 32$ dimensions.

In the third layer, $x^{2}$ is transformed to $x^{3}$, i.e. the FIP features, similar to the second layer, but without pooling.

\footnotetext{
${ }^{1}$ In our notation, $X \in \mathbb{R}^{a, b}$ means $X$ is a two dimensional matrix with $a$ rows and $b$ columns. $x \in \mathbb{R}^{a \times b}$ means $x$ is a vector with $a \times b$ dimensions. Also, $[x ; y]$ means that we concatenate vectors or matrices $x$ and $y$ column-wisely, while $[x y]$ means that we concatenate $x$ and $y$ row-wisely.

${ }^{2}$ For the convolutional neural network such as [14], the non-zero values are the same for each row.

${ }^{3}$ Note that in the conventional deep model [9], there is a bias term $b$, so that the output is $\sigma(W x+b)$. Since $W x+b$ can be written as $\widetilde{W} \widetilde{x}$, we drop the bias term $b$ for simplification.
}

Thus, $x^{3}$ is the same size as $x^{2}$.

$$
x^{3}=\sigma\left(W^{3} x^{2}\right),
$$

where $W^{3}=\left[W_{1}^{3} ; \ldots ; W_{32}^{3}\right], \forall W_{i}^{3} \in \mathbb{R}^{24 \times 24, n_{2}}$ and $x^{2}=$ $\left[x_{1}^{2} ; \ldots ; x_{32}^{2}\right] \in \mathbb{R}^{n_{2}}$, respectively.

Finally, the reconstruction layer transforms the FIP features $x^{3}$ to the frontal face image $y$, through a weight matrix $W^{4} \in \mathbb{R}^{n_{0}, n_{2}}$,

$$
y=\sigma\left(W^{4} x^{3}\right) .
$$

\section{Training}

Training our deep network requires estimating all the weight matrices $\left\{W^{i}\right\}$ as introduced above, which is challenging because of the millions of parameters. Therefore, we first initialize the weights and then update them all. $V^{1}$ and $V^{2}$ are manually defined [13] and fixed.

\subsection{Parameter Initialization}

We cannot employ RBMs [9] to unsupervised pre-train the weight matrices, because our input/output data are in different spaces. Therefore, we devise a supervised method based on the least square dictionary learning. As shown in Fig $3, X^{3}=\left\{x_{i}^{3}\right\}_{i=1}^{m}$ are a set of FIP features and $\bar{Y}=$ $\left\{\bar{y}_{i}\right\}_{i=1}^{m}$ are a set of target images, where $m$ denotes the number of training examples. Our objective is to minimize the reconstruction error

$$
\arg \min _{W^{1}, W^{2}, W^{3}, W^{4}}\left\|\bar{Y}-\sigma\left(W^{4} X^{3}\right)\right\|_{F}^{2},
$$

where $\|\cdot\|_{F}$ is the Frobenius norm. Optimizing Eq 6 is not trivial because of its nonlinearity. However, we can initialize the weight matrices layer-wisely as

$$
\begin{aligned}
& \arg \min _{W^{1}}\left\|\bar{Y}-O W^{1} X^{0}\right\|_{F}^{2}, \\
& \arg \min _{W^{2}}\left\|\bar{Y}-P W^{2} X^{1}\right\|_{F}^{2}, \\
& \arg \min _{W^{3}}\left\|\bar{Y}-Q W^{3} X^{2}\right\|_{F}^{2}, \\
& \quad \arg \min _{W^{4}}\left\|\bar{Y}-W^{4} X^{3}\right\|_{F}^{2} .
\end{aligned}
$$

In Eq77. $X^{0}=\left\{x_{i}^{0}\right\}_{i=1}^{m}$ is a set of input images. $W^{1}$ has been introduced in $\operatorname{Sec} 3$, so that $W^{1} X^{0}$ results in 32 feature maps for each input. $O$ is a fixed binary matrix that sums together the pixels in the same position of these feature maps, which makes $O W^{1} X^{0}$ at the same size as $\bar{Y}$. In Eq $8, X^{1}=\left\{x_{i}^{1}\right\}_{i=1}^{m}$ is a set of outputs of the first locally connected layer before pooling and $P$ is also a fixed binary matrix, which sums together the corresponding pixels and rescales the results to the same size as $\bar{Y} . Q, X^{2}$ in Eq 9 are defined in the same way.

Intuitively, we first directly use $X^{0}$ to approximate $\bar{Y}$ with a linear transform $W^{1}$ without pooling. Once 
$W^{1}$ has been initialized, $X^{1}=V^{1} \sigma\left(W^{1} X^{0}\right)$ is used to approximate $\bar{Y}$ again with another linear transform, $W^{2}$. We repeat this process until all the matrices have been initialized. A similar strategy has been adopted by [33], which learns different levels of representations with a convolutional architecture. All of the above equations have closed-form solutions. For example, $W^{0}=$ $\left(O^{T} O\right)^{-1}\left(O^{T} \bar{Y} X^{0^{T}}\right)\left(X^{0} X^{0^{T}}\right)^{-1}$. The other matrices can be computed in the same way.

\subsection{Parameter Update}

We update all the weight matrices after the initialization by minimizing the loss function of reconstruction error

$$
E\left(X^{0} ; \mathbf{W}\right)=\|\bar{Y}-Y\|_{F}^{2},
$$

where $\mathbf{W}=\left\{W^{1}, \ldots, W^{4}\right\} . X^{0}=\left\{x_{i}^{0}\right\}, \bar{Y}=\left\{\bar{y}_{i}\right\}$, and $Y=\left\{y_{i}\right\}$ are a set of input images, a set of target images, and a set of reconstructed images, respectively. We update $\mathbf{W}$ using the stochastic gradient descent, in which the update rule of $W^{i}, i=1 \ldots 4$, in the $k$-th iteration is

$$
\begin{aligned}
& \Delta_{k+1}=0.9 \cdot \Delta_{k}-0.004 \cdot \epsilon \cdot W_{k}^{i}-\epsilon \cdot \frac{\partial E}{\partial W_{k}^{i}}, \\
& W_{k+1}^{i}=\Delta_{k+1}+W_{k}^{i},
\end{aligned}
$$

where $\Delta$ is the momentum variable [20], $\epsilon$ is the learning rate, and $\frac{\partial E}{\partial W^{i}}=x^{i-1}\left(e^{i}\right)^{T}$ is the derivative, which is computed as the outer product of the back-propagation error $e^{i}$ and the feature of the previous layer $x^{i-1}$. In our deep network, there are three different expressions of $e^{i}$. First, for the transformation layer, $e^{4}$ is computed based on the derivative of the linear rectified function [19]

$$
e_{j}^{4}=\left\{\begin{array}{ll}
{[\bar{y}-y]_{j},} & \delta_{j}^{4}>0 \\
0, & \delta_{j}^{4} \leq 0
\end{array},\right.
$$

where $\delta_{j}^{4}=\left[W^{4} x^{3}\right]_{j} \cdot[\cdot]_{j}$ denotes the $j$-th element of a vector.

Similarly, back-propagation error for $e^{3}$ is computed as

$$
e_{j}^{3}=\left\{\begin{array}{ll}
{\left[W^{4^{T}} e^{4}\right]_{j},} & \delta_{j}^{3}>0 \\
0, & \delta_{j}^{3} \leq 0
\end{array},\right.
$$

where $\delta_{j}^{3}=\left[W^{3} x^{2}\right]_{j}$.

We compute $e^{1}$ and $e^{2}$ in the same way as $e^{3}$ since they both adopt the same activation function. There is a slight difference due to down-sampling. For these two layers, we must up-sample the corresponding back-propagation error $e$ so that it has the same dimensions as the input feature. This strategy has been introduced in [14]. We need to enforce the weight matrices to have locally connected structures after each gradient step as introduced in [12]. We implement this by setting the corresponding matrix elements to zeros, if there supposed to be no connections.

\section{Experiments}

We conduct two sets of experiments. Sec 5.1 compares with state-of-the-art methods and learning-based descriptors. Sec 5.2 demonstrates that classical face recognition methods can be significantly improved when applied on our reconstructed face images in the canonical view.

Dataset. To extensively evaluate our method under different poses and illuminations, we select the MultiPIE face database [7], which contains 754,204 images of 337 identities. Each identity has images captured under 15 poses and 20 illuminations. These images were captured in four sessions during different periods. Like the previous methods [3, 18, 17], we evaluate our algorithm on a subset of the MultiPIE database, where each identity has images from all the four sections under seven poses from yaw angles $-45^{\circ} \sim+45^{\circ}$, and 20 illuminations marked as ID 00-19 in MultiPIE. This subset has 128,940 images.

\subsection{Face Recognition}

The existing works conduct experiments on MultiPIE with three different settings: Setting-I was introduced in [3, 18, 34]; Setting-II and Setting-III were introduced in [17]. We describe these settings below.

Setting-I and Setting-II only adopt images with different poses, but with neutral illumination marked as ID 07 . They evaluate robustness to pose variations. For Setting-I, the images of the first 200 identities in all the four sessions are chosen for training, and the images of the remaining 137 identities for test. During test, one frontal image (i.e. $0^{\circ}$ ) of each identity in the test set is selected to the gallery, so there are 137 gallery images in total. The remaining images from $-45^{\circ} \sim+45^{\circ}$ except $0^{\circ}$ are selected as probes. For Setting-II, only the images in session one are used, which only has 249 identities. The images of the first 100 identities are for training, and the images of the remaining 149 identities for test. During test, one frontal image of each identity in the test set is selected in the gallery. The remaining images from $-45^{\circ} \sim+45^{\circ}$ except $0^{\circ}$ are selected as probes.

Setting-III also adopts images in session one for training and test, but it utilizes the images under all the 7 poses and 20 illuminations. This is to evaluate the robustness when both pose and illumination variations are present. The selection of probes and gallery are the same as Setting-II.

We evaluate both the FIP features and the reconstructed images using the above three settings. Face images are roughly aligned according to the positions of eyes, and rescaled to $96 \times 96$. They are converted to grayscale images. The mean value over the training set is subtracted from each pixel. For each identity, we use the images with 6 poses ranging from $-45^{\circ} \sim+45^{\circ}$ except $0^{\circ}$, and 19 illuminations marked as ID $00-19$ except 07 , as input to train our deep network. The reconstruction target is the 


\begin{tabular}{lcccccccc}
\hline & $-45^{\circ}$ & $-30^{\circ}$ & $-15^{\circ}$ & $+15^{\circ}$ & $+30^{\circ}$ & $+45^{\circ}$ & Avg & Pose \\
\hline LGBP[34] & 37.7 & 62.5 & 77 & 83 & 59.2 & 36.1 & 59.3 & $\checkmark$ \\
VAAM[3] & 74.1 & 91 & 95.7 & 95.7 & 89.5 & 74.8 & 86.9 & $\checkmark$ \\
FA-EGFC[18] & 84.7 & 95 & 99.3 & 99 & 92.9 & 85.2 & 92.7 & $\times$ \\
SA-EGFC[18] & 93 & $\mathbf{9 8 . 7}$ & 99.7 & $\mathbf{9 9 . 7}$ & $\mathbf{9 8 . 3}$ & $\mathbf{9 3 . 6}$ & $\mathbf{9 7 . 2}$ & $\checkmark$ \\
\hline LE[4]+LDA & 86.9 & 95.5 & 99.9 & $\mathbf{9 9 . 7}$ & 95.5 & 81.8 & 93.2 & $\times$ \\
CRBM[10]+LDA & 80.3 & 90.5 & 94.9 & 96.4 & 88.3 & 75.2 & 87.6 & $\times$ \\
\hline FIP+LDA & $\mathbf{9 3 . 4}$ & 95.6 & $\mathbf{1 0 0 . 0}$ & 98.5 & 96.4 & 89.8 & 95.6 & $\times$ \\
RL+LDA & $\mathbf{9 5 . 6}$ & $\mathbf{9 8 . 5}$ & $\mathbf{1 0 0 . 0}$ & 99.3 & $\mathbf{9 8 . 5}$ & $\mathbf{9 7 . 8}$ & $\mathbf{9 8 . 3}$ & $\times$ \\
\hline
\end{tabular}

Table 1. Recognition rates under Setting-I. The first and the second highest rates are highlighted. " $\checkmark$ " indicates the method needs to know the pose; " $\times$ ", otherwise.

\begin{tabular}{lcccccccc}
\hline & $-45^{\circ}$ & $-30^{\circ}$ & $-15^{\circ}$ & $+15^{\circ}$ & $+30^{\circ}$ & $+45^{\circ}$ & Avg & Pose \\
\hline $\mathrm{LE}[4]+\ell_{2}$ & 63.0 & $\mathbf{9 0 . 0}$ & $\mathbf{9 5 . 0}$ & 95.0 & 90.0 & 61.5 & 82.4 & $\times$ \\
$\mathrm{CRBM}[10]+\ell_{2}$ & 59.9 & 68.5 & 94.9 & 83.2 & 88.3 & 66.4 & 74.7 & $\times$ \\
\hline $\mathrm{FIP}+\ell_{2}$ & $\mathbf{7 8 . 6}$ & 87.9 & 94.9 & $\mathbf{9 6 . 1}$ & $\mathbf{9 1 . 8}$ & $\mathbf{8 0 . 8}$ & $\mathbf{8 8 . 3}$ & $\times$ \\
$\mathrm{RL}+\ell_{2}$ & $\mathbf{9 4 . 9}$ & $\mathbf{9 4 . 2}$ & $\mathbf{9 8 . 5}$ & $\mathbf{9 9 . 3}$ & $\mathbf{9 8 . 5}$ & $\mathbf{8 4 . 0}$ & $\mathbf{9 4 . 9}$ & $\times$ \\
\hline
\end{tabular}

Table 2. Recognition rates under Setting-I. The proposed features are compared with LE and CRBM using only the $\ell_{2}$ distance for face recognition. The first and the second highest rates are highlighted. " $\checkmark$ " indicates the method needs to know the pose; " $\times$ ”, otherwise.

image captured in $0^{\circ}$ under neutral illumination (ID 07 ). In the test stage, in order to better demonstrate the proposed methods, we directly adopt the FIP and the reconstructed images (denoted as RL) as features for face recognition.

\subsubsection{Results of Setting-I}

In this setting, we show superior results in Table 1, where the FIP and RL features are compared with four methods, including LGBP [34], VAAM [3], FA-EGFC [18], and SAEGFC [18], and two learning-based descriptors, including LE [4] and CRBM [10]. As discussed in Sec 1, LGBP is a 2D-based method, while VAAM, FA-EGFC, and SAEGFC used 3D face models. We apply LDA on LE, CRBM, FIP, and RL to obtain compact features. Note that LGBP, VAAM, and SA-EGFC need to know the pose of a probe, which means that they build different models to account for different poses specifically. We do not need to know the pose of the probe, since our deep network can extract FIP features and reconstruct the face image in the canonical view given a probe under any pose and any illumination. This is one of our advantages over existing methods.

Several observations can be made from Table 11. First, $\mathrm{RL}$ performs best on the averaged recognition rates and five poses. The improvement is larger for larger pose variations. It is interesting to note that RL even outperforms all the 3D-based models, which verifies that our reconstructed face images in the canonical view are of high quality and robust to pose changes. Fig 4 shows several reconstructed images, indicating that RL can effectively remove the variations of poses and illuminations, while still retains the intrinsic shapes and structures of the identities.

\begin{tabular}{lcccccccc}
\hline & $-45^{\circ}$ & $-30^{\circ}$ & $-15^{\circ}$ & $+15^{\circ}$ & $+30^{\circ}$ & $+45^{\circ}$ & Avg & Pose \\
\hline Li [17] & 97.0 & 97.0 & $\mathbf{1 0 0 . 0}$ & $\mathbf{1 0 0 . 0}$ & 97.0 & 92.0 & 96.8 & $\checkmark$ \\
RL+LDA & $\mathbf{9 7 . 8}$ & $\mathbf{9 8 . 6}$ & $\mathbf{1 0 0 . 0}$ & $\mathbf{1 0 0 . 0}$ & $\mathbf{9 8 . 6}$ & $\mathbf{9 8 . 4}$ & $\mathbf{9 8 . 4}$ & $\times$ \\
\hline
\end{tabular}

Table 3. Recognition rates of RL+LDA compared with Li [17] under Setting-II. " $\checkmark$ " indicates the method needs to know the pose; " $\times$ ", otherwise.

\begin{tabular}{|c|c|c|c|c|c|c|c|c|}
\hline & \multicolumn{8}{|c|}{ Recognition Rates on Different Poses } \\
\hline & $-45^{\circ}$ & $-30^{\circ}$ & $-15^{\circ}$ & $+15^{\circ}$ & $+30^{\circ}$ & $+45^{\circ}$ & Avg & Pose \\
\hline $\operatorname{Li}[17]$ & 63.5 & 69.3 & 79.7 & 75.6 & 71.6 & 54.6 & 69.3 & $\checkmark$ \\
\hline RL+LDA & 67.1 & 74.6 & 86.1 & 83.3 & 75.3 & 61.8 & 74.7 & $x$ \\
\hline \multicolumn{9}{|c|}{ Recognition Rates on Different Illuminations } \\
\hline & 00 & 01 & 02 & 03 & 0 & & 05 & 06 \\
\hline $\operatorname{Li}[17]$ & 51.5 & 49.2 & 55.7 & 62. & 79 & & 88.3 & 97.5 \\
\hline \multirow[t]{2}{*}{$\mathrm{RL}+\mathrm{LDA}$} & 72.8 & 75.8 & 75.8 & 75. & 75 & & 75.7 & 75.7 \\
\hline & 08 & 09 & 10 & 11 & 1 & & 13 & 14 \\
\hline Li [17] & 97.7 & 91.0 & 79.0 & 64. & 54 & & 47.7 & 67.3 \\
\hline \multirow[t]{2}{*}{$\mathrm{RL}+\mathrm{LDA}$} & 75.7 & 75.7 & 75.7 & 75. & 75 & & 75.7 & 73.4 \\
\hline & 15 & 16 & 17 & 18 & 1 & 9 & \multicolumn{2}{|c|}{ Avg. } \\
\hline Li [17] & 67.7 & 75.5 & 69.5 & 67. & 50 & 0.8 & \multicolumn{2}{|c|}{69.3} \\
\hline $\mathrm{RL}+\mathrm{LDA}$ & 73.4 & 73.4 & 73.4 & 72. & 72 & 2.9 & \multicolumn{2}{|c|}{74.7} \\
\hline
\end{tabular}

Table 4. Recognition rates of RL+LDA compared with Li [17] under Setting-III. " $\checkmark$ " indicates the method needs to know the pose; " $\times$ ", otherwise.

Second, FIP features are better than the two learningbased descriptors and the other three methods except SAEGFC, which used the 3D model and required the pose of the probe. We further report the results of FIP compared with LE and CRBM using only $\ell_{2}$ distance in Table 2 . The RL and FIP outperform the above two learning based features, especially when large pose variations are present.

Third, although FIP does not exceed RL, its still a valuable representation, because it has the sparse property and can reconstruct RL efficiently and losslessly.

\subsubsection{Results of Setting-II and Setting-III}

Li et al. [17] evaluated on these two settings and reported the state-of-the-art results. Setting-II covers only pose variations and Setting-III covers both pose and illumination variations.

For Setting-II, the results of RL+LDA compared with [17] are reported in Table 3, which shows that RL obtains the best results on all the poses. Note that the poses of probes in [17] are assumed to be given, which means they trained a different model for each pose separately. [17] did not report detailed recognition rates when the poses of the probes are unknown, except for describing a $20-30 \%$ decline of the overall recognition rate.

For Setting-III, RL+LDA is compared with [17] on images with both pose and illumination variations. Table 4 reports that our approach achieves better results on all the poses and illuminations. The recognition rate under a pose is the averaged result over all the possible illuminations. Similarly, the recognition rate under one illumination 
condition is the averaged result of all the possible poses. We observe that the performance of RL+LDA under different illuminations is close because RL can well remove the effect of different types of illuminations.

\subsection{Improve Classical Face Recognition Methods}

In this section, we will show that the conventional feature extraction and dimension reduction methods in the face recognition literature, such as LBP [2], Gabor [31], PCA [11], LDA [1], and Sparse Coding (SC) [32], can achieve significant improvement when they adopt our reconstructed images as input.

We conduct three experiments using the training/testing data of Setting-I. First, we show the advantage of our reconstructed images in the canonical view over the original images. Second, we show the improvements of Gabor when it is extracted on our reconstructed images. Third, we show that LBP can be improved as well.

In the first experiment, $\ell_{2}$ distance, SC, PCA, LDA, and PCA+LDA are directly applied on the raw pixels of the original images and our reconstructed images, respectively. The recognition rates are reported in Fig[5(a) where the results on the original images and the reconstructed images are illustrated as solid bars (front) and hollow bars (back). We observe that each of the above methods can be improved at least $30 \%$ on average. They can achieve relatively high performance on different poses, because our reconstruction layer can successfully recover the frontal face image. For example, the recognition rates of SC on different poses using the original images are $20.9 \%, 43.6 \%, 65.0 \%, 66.1 \%$, $38.3 \%$, and $26.9 \%$, respectively, while $92.7 \%, 97.1 \%$, $97.8 \%, 98.5 \%, 97.8 \%$, and $81.8 \%$, respectively, using the reconstructed images.

In the second experiment, we extract Gabor features on both the original images and reconstructed images. We observe large improvements by using the reconstructed images. Specifically, for each image in $96 \times 96$, we evenly select $11 \times 10$ keypoints and apply 40 Gabor kernels $(5$ scales $\times 8$ orientations) on each of these keypoints. We again use the $\ell_{2}$ distance, PCA, LDA, and PCA+LDA for face recognition. The results are shown in Fig 5(b),

In the third experiment, we extract LBP features on both original images and reconstructed images. Specifically, we divide each $96 \times 96$ image into $12 \times 12$ cells, and the 59 uniform binary patterns are computed in each cell. We then adopt the $\chi^{2}$ distance, PCA, LDA, and PCA+LDA for face recognition. Fig 5(c) shows that LBP combined with all these methods can also be significantly improved. For instance, the averaged recognition rate of $\mathrm{LBP}+\chi^{2}$ using the original images is $75.9 \%$, and the corresponding accuracy on our reconstructed images, i.e. $\mathrm{RL}+\mathrm{LBP}+\chi^{2}$, is $96.5 \%$, which is better than $94.9 \%$ of $R L+\ell_{2}$ in Table 2 .

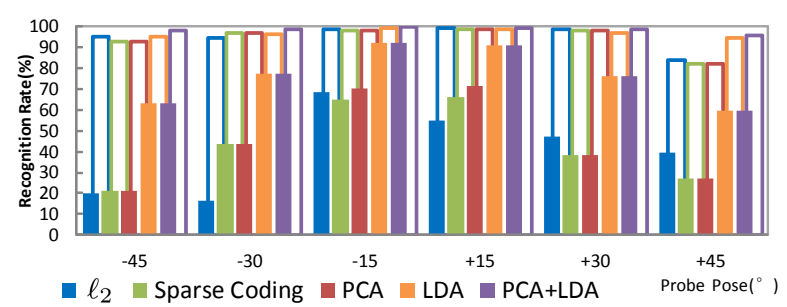

(a) Pixels

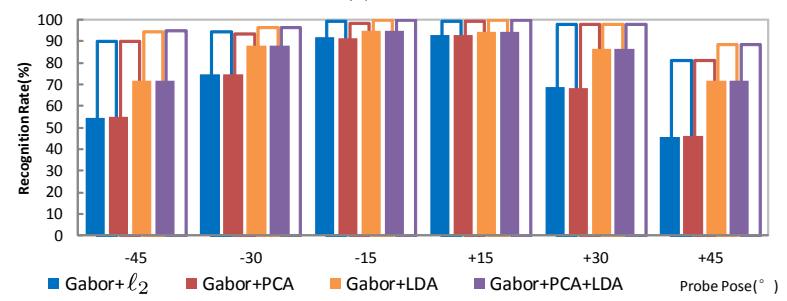

(b) Gabor descriptors

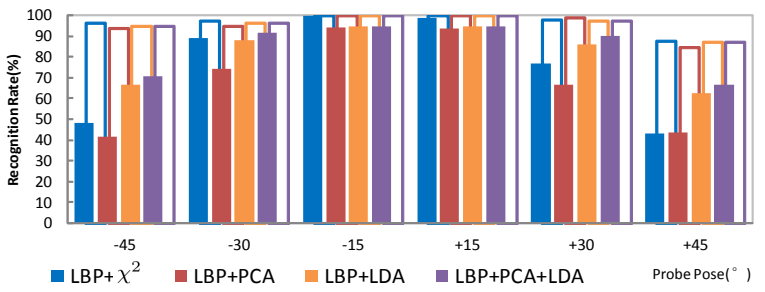

(c) LBP descriptors

Figure 5. The conventional face recognition methods can be improved when they are applied on our reconstructed images. The results of three descriptors (pixel intensity, Gabor, and LBP) and four face recognition methods ( $\ell_{2}$ or $\chi^{2}$ distance, sparse coding (SC), PCA, and LDA) are reported in (a), (b) and (c), respectively. The hollow bars are the performance of these methods applied on our reconstructed images, while the solid bars are on the original images.

\section{Conclusion}

We have proposed identity-preserving features for face recognition. The FIP features are not only robust to pose and illumination variations, but can also be used to reconstruct face images in the canonical view. FIP is learned using a deep model that contains feature extraction layers and a reconstruction layer. We show that FIP features outperform the state-of-the-art face recognition methods. We have aslo improved classical face recognition methods by applying them on our reconstructed face images. In the future work, we will extend the framework to deal with robust face recognition in other difficult conditions such as expression change and face sketch recognition [25, 30], and will combine FIP features with more classic face recognition approaches to further improve the performance [28, 29, 27].

\section{References}

[1] H. Abdi. Discriminant correspondence analysis. Encyclopedia of Measurement and Statistics, 2007.

[2] T. Ahonen, A. Hadid, and M. Pietikainen. Face description with local binary patterns: Application to face recognition. IEEE Transactions on Pattern Analysis and Machine Intelligence, 28(12):2037-2041, 2006. 

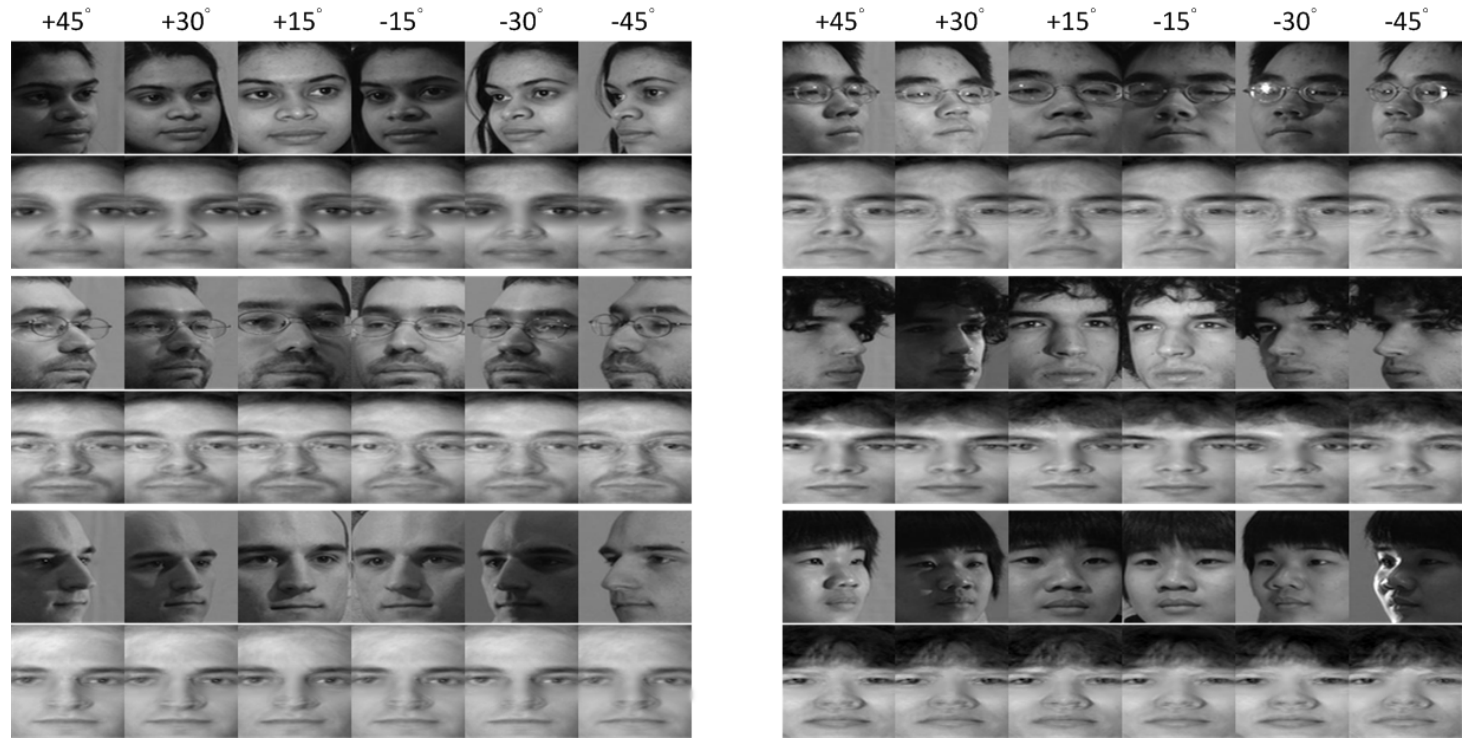

Figure 4. Examples of face reconstruction. For each identity, we select its images with 6 poses and arbitrary illuminations. The reconstructed frontal face images under neutral illumination are visualized below. We clearly see that our method can remove the effects of both poses and illuminations, and retains the intrinsic face shapes and structures of the identity.

[3] A. Asthana, T. K. Marks, M. J. Jones, K. H. Tieu, and M. Rohith. Fully automatic pose-invariant face recognition via $3 \mathrm{~d}$ pose normalization. In $I C C V$, 2011

[4] Z. Cao, Q. Yin, X. Tang, and J. Sun. Face recognition with learning-based descriptor. In $C V P R, 2010$.

[5] C. D. Castillo and D. W. Jacobs. Wide-baseline stereo for face recognition with large pose variation. In $C V P R, 2011$.

[6] S. Chopra, R. Hadsell, and Y. LeCun. Learning a similarity metric discriminatively, with application to face. In $C V P R, 2005$.

[7] R. Gross, I. Matthews, J. Cohn, T. Kanade, and S. Baker. Multi-pie. In International Conference on Automatic Face and Gesture Recognition, 2008.

[8] Y. Guo, G. Zhao, M. Pietikainen, and Z. Xu. Descriptor learning based on fisher separation criterion for texture classification. In ACCV, 2010.

[9] G. E. Hinton, S. Osindero, and Y.-W. Teh. A fast learning algorithm for deep belief nets. Neural Computation, 18(7):1527-1554, 2006.

[10] G. B. Huang, H. Lee, and E. Learned-Miller. Learning hierarchical representations for face verification with convolutional deep belief networks. In CVPR, 2012.

[11] I. T. Jolliffe. Principal component analysis, volume 487. 1986.

[12] A. Krizhevsky, I. Sutskever, and G. Hinton. Imagenet classification with deep convolutional neural networks. In NIPS, 2012.

[13] Q. V. Le, J. Ngiam, Z. Chen, D. Chia, P. W. Koh, and A. Y. Ng. Tiled convolutional neural networks. In NIPS, 2010.

[14] Y. LeCun, L. Bottou, Y. Bengio, and P. Haffner. Gradient-based learning applied to document recognition. In Proceedings of the IEEE, 1998.

[15] H. Lee, R. Grosse, R. Ranganath, and A. Y. Ng. Convolutional deep belief networks for scalable unsupervised learning of hierarchical representations. In Proc. 26th International Conference on Machine Learning, pages 609-616. ACM, 2009.

[16] Z. Lei, D. Yi, and S. Z. Li. Discriminant image filter learning for face recognition with local binary pattern like representation. In CVPR, 2012.

[17] A. Li, S. Shan, and W. Gao. Coupled bias-variance tradeoff for cross-pose face recognition. IEEE Transactions on Image Processing, 21(1):305-315, 2012.

[18] S. Li, X. Liu, X. Chai, H. Zhang, S. Lao, and S. Shan. Morphable displacement field based image matching for face recognition across pose. In ECCV. 2012.

[19] V. Nair and G. E. Hinton. Rectified linear units improve restricted boltzmann machines. In Proc. 27th International Conference on Machine Learning, 2010.

[20] N. Qian. On the momentum term in gradient descent learning algorithms. Neural Networks, 1999.

[21] M. Ranzato, J. Susskind, V. Mnih, and G. Hinton. On deep generative models with applications to recognition. In CVPR, 2011.
[22] R. Salakhutdinov and G. E. Hinton. Deep boltzmann machines. In Proceedings of the International Conference on Artificial Intelligence and Statistics, volume 5, pages 448-455, 2009.

[23] F. Schroff, T. Treibitz, D. Kriegman, and S. Belongie. Pose, illumination and expression invariant pairwise face-similarity measure via doppelgänger list comparison. In ICCV, 2011.

[24] Y. Sun, X. Wang, and X. Tang. Hybrid deep learning for face verification. In ICCV, 2013.

[25] X. Tang and X. Wang. Face sketch recognition. IEEE Transactions on Circuits and Systems for Video Technology, 14(1):50-57, 2004.

[26] A. Wagner, J. Wright, A. Ganesh, Z. Zhou, H. Mobahi, and Y. Ma. Toward a practical face recognition system: Robust alignment and illumination by sparse representation. IEEE Transactions on Pattern Analysis and Machine Intelligence, 34(2):372-386, 2012.

[27] X. Wang and X. Tang. Dual-space linear discriminant analysis for face recognition. In $C V P R, 2004$.

[28] X. Wang and X. Tang. A unified framework for subspace face recognition. IEEE Transactions on Pattern Analysis and Machine Intelligence, 26(9):12221228, 2004.

[29] X. Wang and X. Tang. Random sampling for subspace face recognition. International Journal of Computer Vision, 70(1):91-104, 2006.

[30] X. Wang and X. Tang. Face photo-sketch synthesis and recognition. IEEE Transactions on Pattern Analysis and Machine Intelligence, 31(11):1955-1967, 2009.

[31] L. Wiskott, J.-M. Fellous, N. Kuiger, and C. von der Malsburg. Face recognition by elastic bunch graph matching. IEEE Transactions on Pattern Analysis and Machine Intelligence, 19(7):775-779, 1997.

[32] J. Wright, A. Y. Yang, A. Ganesh, S. S. Sastry, and Y. Ma. Robust face recognition via sparse representation. IEEE Transactions on Pattern Analysis and Machine Intelligence, 31(2):210-227, 2009.

[33] M. D. Zeiler, G. W. Taylor, and R. Fergus. Adaptive deconvolutional networks for mid and high level feature learning. In ICCV, 2011.

[34] W. Zhang, S. Shan, W. Gao, X. Chen, and H. Zhang. Local gabor binary pattern histogram sequence (lgbphs): A novel non-statistical model for face representation and recognition. In ICCV, 2005.

[35] W. Zhang, X. Wang, and X. Tang. Coupled information-theoretic encoding for face photo-sketch recognition. In CVPR, 2011.

[36] X. Zhang and Y. Gao. Face recognition across pose: A review. Pattern Recognition, 42(11):2876-2896, 2009. 